\title{
Acknowledgment to Reviewers of Social Sciences in 2020
}

\author{
Social Sciences Editorial Office
}

Citation: Social Sciences Editorial Office. 2021. Acknowledgment to Reviewers of Social Sciences in 2020. Social Sciences 10: 30. https://doi.org/ 10.3390/socsci10020030

Published: 20 January 2021

Publisher's Note: MDPI stays neutral with regard to jurisdictional claims in published maps and institutional affiliations.

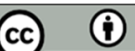

Copyright: @ 2021 by the author. Licensee MDPI, Basel, Switzerland. This article is an open access article distributed under the terms and conditions of the Creative Commons Attribution (CC BY) license (http://creativecommons.org/licenses/by/4.0/).

MDPI AG, St. Alban-Anlage 66, 4052 Basel, Switzerland

Peer review is the driving force of journal development, and reviewers are gatekeepers who ensure that Social Sciences maintains its standards for the high quality of its published papers. Thanks to the cooperation of our reviewers, in 2020, the median time to first decision was 18 days and the median time to publication was 44 days. The editors would like to express their sincere gratitude to the following reviewers for their precious time and dedication, regardless of whether the papers were finally published:

Aarnio-Linnanvuori, Essi

Abduljaber, Malek

Abel, Gillian

Abreu, António

Aceytuno, María-Teresa

Adu-Gyamfi, Jones

Aguaded-Ramírez, Eva

Agudo Romeo, María Del Mar

Aguilar, Lourdes

Aiello, Emilia

Alcaraz Carrillo De Albornoz, Vicente

Alghamdi, Abdulaziz

Aliu, Armando

Allen, Katherine

Alonso Centeno, Almudena

Alrahaili, Musaad

Alvarez-Sousa, Antonio

Amante, Ana

Amitrano, Cristina Caterina

Andell, Paul

Anderson, Charity

Anderson, Joel

Andreassen, Pernille

Andreatta, Daniela

Andrew, Lesley

Anson, April

Appe, Susan

Aptekar, Sofya

Arcangeli, Giulio

Arcila Calderón, Carlos

Arias, Ana Victoria

Arnold, Bruce Baer

Arribas Galagarra, Silvia

Asakura, Takashi
Babcock, Rebecca

Baer, Judith C.

Bagga-Gupta, Sangeeta

Bagley, Christopher Adam

Bakali, Naved

Baldwin, John

Ballesteros, Alfonso

Bándy, Katalin

Banh, Jenny

Bansah, Kenneth

Barak, Gregg

Barbee, Harry

Bartkowiak-Theron, Isabelle

Bartkowski, John

Bartniczak, Bartosz

Baumgarth, William P.

Beck, Krzysztof

Behtoui, Alireza

Bejaković, Predrag

Belcher, John R.

Belford, Nish

Belmonte, Jesús López

Belmonte-Ureña, Luis J.

Benlloch Osuna, Mayte

Bennachie, Calum

Benton, J. Edwin

Bereményi, Bálint Ábel

Berescu, Catalin

Berezuk, André Geraldo

Bergersen, Ane

Bernal-Bravo, César

Berntzen, Lasse

Berrick, Jill

Berthold, Anne 
Bezruchka, Stephen

Bian, Yanjie

Bianco, Rosella

Bichler, Gisela

Bielinis, Ernest

Bielski, Stanisław

Binti Ramli, Nur Suhaili

Blanco Rivero, Jose Javier

Blanco, Julien

Blaško, Rudolf

Blašková, Martina

Boardman, Jed

Bockerman, Petri

Bocsi, Veronika

Bolotov, Ilya

Bolz, Daphné

Bontje, Marco

Born, Christopher

Borzikowsky, Christoph

Bostenaru Dan, Maria

Bovan, Kosta

Bradley, Harriet

Brand, Serge

Bresin, Konrad

Brisman, Avi

Brokalaki, Zafeirenia

Brown, Kenly

Brown, Natalie

Broxmeyer, Jeffrey

Brzoska, Michael

Buckley, Geoffrey

Bueno, Salvador

Buessing, Alexander

Bugaj, Justyna Maria

Bungau, Constantin

Bush, Judy

Butler-Henderson, Kerryn

Butters, Andrew

Caballero, Rafael Repiso

Caccia, Federico Carril

Caeiro-Rodríguez, Manuel

Calabuig-Moreno, Ferran

Camarero-Figuerola, Marta

Camerino, Donatella

Campbell, Rochelle

Campion, Kristy

Cañas, Elizabeth

Cannas Da Silva, Luisa

Cannito, Maddalena

Canosa, Antonia

CAO, Wangnan
Caranta, Roberto

Carausu, E. M.

Card, Kiffer

Carreri, Anna

Carr-Harris, Andrew

Carrillo, Irene

Carter, Niambi M.

Cartmel, Jennifer

Castañeda, Ernesto

Castellví, Jordi

Castro, Miguel

Cefalo, Ruggero

Cerejo, Dalila

Cerniglia, Luca

Certomà, Chiara

Cervera-Garvi, Pablo

Cervi, Laura

Chaljub, Jeanette

Chang, Po-Ju

Chapman, Andrew John

Chapple, Simon

Chen, Weizhi

Chiew Hong, Ng

Chirico, Francesco

Chistolini, Sandra

Choi, Pak-Sing

Christoff, Peg

Cierniak-Emerych, Anna

Cieślińska, Barbara

Civero, Gennaro

Claessens, Brigitte

Clawson, Rachael

Clisby, Suzanne

Cobelli, Nicola

Cole, Matthew

Conversi, Daniele

Cook, Simon

Cooke, Barbara

Corkindale, David

Cornelius Smith, Erika

Costa, Bruno

Cottone, Paolo

Crawford, Joseph

CRISTEA, MIRELA

Crow, Graham

Cunha, Jorge

Cunitz, Katrin

Cunningham, Rebecca

Cutrona, Carolyn

Dąbrowski, Karol

Dalmau, Antoni 
Dantas, Carina

Dasandi, Niheer

Dasgupta, Satarupa

De Graaff, Erik

Del Espino-Díaz, Luis

Delgado, Paulo

Dellinger, Myanna

Densley, James

Denton, Julie Anne

DeRose, Laurie Fields

Dettori, Marco

DeVance Taliaferro, Jocelyn

Devaney, Carmel

Dhir, Amandeep

Di Battista, Silvia

Di Iacovo, Francesco

Diaconeasa, Maria Claudia

Díaz- Noci, Javier

Díaz, Mónica

Diogo, Elisete

Dos Santos, Luis Miguel

Drechselová, Lucie

Dunbar, Angel S.

Dundes, Lauren

Dwyer, Angela

Dziuba, Szymon

Earnshaw, Sarah

Elasra, Amira

Elias, Anwen

Elkordy, Angela

Ellefson, Michelle

Elvira, Nica

Ennaji, Moha

Epting, Shane

Erceg, Aleksandar

Erenli, Kai

Eriksson, Anna

Esnard, Talia Randa

Espluga, Josep

Esteban Salvador, Maria Luisa

Estellés, Marta

Evans, Robert

Everitt, Julia

Eversole, Robyn

Faria, Rita

Farrand, Kathleen

Farrelly, Daniel

Feltrero, Roberto

Fernandes, Emília

Fernandez Salinero, Samuel

Fernández, Sylvia
Fernández-Antelo, Inmaculada

Fernández-Prados, Juan Sebastián

Fernández-Revelles, Andrés B.

Ferraz, Hélder

Ferreira, Gil

Ferreira, Jorge

Finnegan, Ciara

Fischer, Karla

Fisher, John

Flanders-Crosby, Jill

Flecha, Ramon

Fleisher, Mark

Fleisher, Mark S.

Flores-Aranda, Jorge

Fordham, Benjamin

Forte, Fabiana

Forte, Pedro

Foust, Regan

Fox, Diana

Frideres, James

Friedman-Sokuler, Naomi

Friesen, John

Friso, Valeria

Frost, Nick

Frumkin, Bruce

Fucà, Romina

Fuentes-Loureiro, María-Ángeles

Fukumoto, Rui

Fung, Sai Fu

Gaggi, Ombretta

Gambini, Annastella

Gamble, Jeffrey Hugh

García-Garnica, Marina

García-Ruiz, Carmen Rosa

Garipov, Ruslan

Gayardon, Ariane De

Geck, Angela

Gedvilaite, Dainora

Gee, Malcolm

Gharehgozli, Orkideh

Gheorghe, Huza

Giannakas, Filippos

Gierczyk, Marcin

Gierycz, Michał

Gilbert, Alan

Glasgow, Trevin

Glynn, Peter J.

Godfrey, Barry

Gómez García, Gerardo

Gómez-García, Melchor

Gómez-Urquiza, Jose L. 
Gonçalves, Rui Abrunhosa

Goniewicz, Krzysztof

Gonzalez Lopez, Maria Jose

González, Rebeca Cristina López

Goodarzi, Mohsen

Gora, Ana Alexandra

Goyes, David Rodríguez

Graham, William

Gray, Peter B.

Greenberg, Jeff

Greene, Zachary

Gregor, Milos

Gregurović, Margareta

Griffin, Susan

Griffiths, Laura

Grigoryan, Lusine

Grindlay, Alejandro

Gromkowska-Melosik, Agnieszka

Guenther, John

Guijo, Valeriana

Gurko, Tatiana

Gutiérrez Ortega, Mónica

Guyer, Joshua J.

Hall, Matthew

Hamed, Moussa Boumadan

Hanada, Shingo

Hanna, Michele

Hardy, Keiran

Harris, Jasmine

Hattingh, Marie

Haynes, Jeffrey

HEFFERNAN, KEVIN

Heiland, Stefan

Henderson, Carol

Hergueta Covacho, Elisa

Herman, Emilia

Hernandez Fernandez, Antonio

Hernández, Antonio Fernández

Herreros, Francisco

Hill, Shirley A.

Hillman, Arye L.

Hintjens, Helen

Hobson, Charles

Hogue, Lawrence

Hołuj, Artur

Hörnsten, Åsa

Horwitz, Ilana

Hovis, Ciara L.

Howell, Jayne

Hribar, Mateja Šmid

Hubbard, Phil
Hugelius, Karin

Hughes, Bryce

Humble, Steve

Hyslop, Ian

Ianos, Maria Gratiela

Iio, Jun

Isaac, Mathew S.

Islam, Farah

Jackson, Kathy Merlock

Jackson, Richard

Jalal, Ahmad

James, Spencer

Jan, Shazia K.

Jarman, Ben

Jeddi Yeganeh, Armin

Jeffrey, Leslie

Jeliazkova, Margarita

Jennings, George

Jensen, Kari B.

Jensen, Michael

Jiménez, Lucía

Jiménez-Eguizabal, Juan Alfredo

Jobe, Alison

Jochnowitz, Leona Deborah

JORGE-VÁZQUEZ, JAVIER

José Sousa, Maria

Jowsey, Tanisha

Judit, Oláh

Juffer, Femmie

Jukneviciene, Vita

Jungbauer-Gans, Monika

Jurado De Los Santos, Pedro

Jurs, Pavel

Kaliszewski, Adam

Kaltsas, Spyridon

Kamenidou, Irene

Kang, Daekook

Kang, Seung-Wan

Karakašić, Mirko

Karampelas, Konstantinos

Karandikar, Sharvari

Karavasilis, Lazaros

Katsantonis, Ioannis G.

Katz, Ilan

Kempadoo, Dr Kemale

Ken, Ivy

Ketels, Christian

Kevin, Dougherty

Keyton, Joann

Kim, Dongshin

Kim, Jong In 
Kim, Namhyun

Kind, Nina

Kirkegaard, Emil O. W.

Kitagawa, Kaori

Klimek, Libor

Klizienè, Irina

Knight, Thomas Daniel

Ko, Hsin-Chi

Konstantinov, Vsevolod

Koos, Agnes

Kopinska, Violetta

Korac-Sanderson, Maja

Koren, Ore

Koura, Fatima

Kovačić, Marko

Kowalewski, Maciej

Krajcsák, Zoltán

Krasny, Marianne

Krolikowska, Karolina

Krysta, Krzysztof

Kuklina, Vera

Kyere, Eric

Kyriakopoulos, Grigorios

Lakatos, Elena Simina

Lamb, David S.

Lambert, Michael

Lambert, Sharon F.

Lammi-Taskula, Johanna

Landsem, Inger

Lanfranchi, Andrea

Langlais, Mickey

LaRue, Robert

Laurentsyeva, Nadzeya

Lawrence, Jeanette A.

Layne, Linda

Leath, Seanna

Lee, David

Lee, Elsa

Lee, Jeong-hoon

Lee, Juheon

Lee, Jui-Hsiang

Leitão, João

Leiva Olivencia, Juan J.

León-Gómez, Brenda Biaani

León-Rubio, José María

Lesyk, Lilia

Lewis, Consuella

Li, Frank

Li, Long

Li, Manyu

Li, Xingwei
Limbocker, Scott

Lindgren, Wrenn Yennie

Lipowski, Mariusz

Liu, Sze Y.

Lloret, Antonio

Logan, John

Longo, Maria Cristina

López-Cordero, Rafael

López-Peláez, Antonio

Löther, Andrea

Lucas, Jack

Luong, Hai

Łuszczki, Edyta

Lyon-Callo, Vincent

Maat, Sekhmet Ra Em Kht

Machnik, Aleksandra

Maftei, Alexandra

Mahdu, Omchand

Majchrzak-Lepczyk, Justyna

Majic, Samantha

Makarovič, Matej

Malega, Ron

Mallion, Jaimee

Malmquist, Anna

Manasia, Loredana

Manca, Stefania

Maniou, Theodora

Mantzaris, Alexander V.

Marbaniang, Domenic

Marcassa, Stefania

Marcellini, Anne

Marín Lladó, Carles

Marín-Díaz, Verónica

Marín-Marín, José-Antonio

Markofski, Wes

Marques Martins, Paula Cristina

Martín, J.E. Jamal

Martínez-López, José Ángel

Martinho, Teresa Duarte

Martos Martínez, África

Mascherini, Gabriele

Mather, Carey

Matikainen, Pasi

Matthews, Todd L.

Mattsson, Jan Christer

Maylor, Uvanney

Mazur, Joanna

McClanahan, Bill

Melidoro, Domenico

Metcalf, Jacob

Meunier, Etienne 
Meyer, Jonas

Miglionico, Andrea

Migozzi, Julien

Mihálik, Jaroslav

Mikhailovich, Katja

Milichovský, František

Milovanović, Ivana

Mims, Lauren

Minassians, Henrik P.

Mingboupha, Nathalie

Minor, Elizabeth Covay

Mitchell, Gregory

Mitroiu, Simona

Mohedano, Félix Ortega

Mokuria, Vicki G.

Molero Jurado, María Del Mar

Molho, Jeremie

Monnot, Christophe

Montagnino, Fabio Maria

Mora-Garcia, Raul-Tomas

Morales, Pablo

Moral-Moral, María

Moreno-Rodriguez, Ricardo

Moriyama, Nobuaki

Morrison, Todd G.

Mortlock, Anita

Mosler, Saruhan

Mugabi, Ivan K.

Mukuni, Joseph

Mulia, Nina

Mullins, Christopher W.

Muñoz, Isidro Sepulveda

Muñoz-Fernández, Noelia

Munson, April

Murphy, Raegan

Mušanović, Jelena

Náñez Alonso, Sergio Luis

Napal, María

Nathans, Laura

Naumov, Nikola

Nemeth, Lynne S.

Neocleous, Gregory

Newaz, Mohammad

Ng Sau Wah, Sarah

Niemistö, Charlotta

Nita, Andreea

Nkhoma, Pearson

Nordtveit, Ernst

Novak, Ken

Nunes, Ana Isabel

O'Leary, Simon
Okubo, Yuko

OLATUNBOSUN, ADENIYI

Oleson, James C.

Olivares, Fernando

Olmos Gómez, María Del Carmen

Opara, Ijeoma

Opasina, Oladapo Kayode

Oprsal, Zdenek

Oropesa Ruiz, Nieves Fátima

Ortega-Sánchez, Delfín

Osanami Törngren, Sayaka

Oselin, Sharon S.

Oswald, John

Ottemo, Andreas

Outten, H. Robert

Owen, Stephen

Owusu-Daaku, Kwame

Paci, Chris

Padrós Cuxart, Maria

Paisal, Marc Salat

Paliszkiewicz, Joanna

Palos, Ana Cristina

Palos-Sánchez, Pedro R.

Papadopoulou, Agnes

Parejo Llanos, Jose Luis

Park, Chang Sup

Partington, Hazel

Passantino, Annamaria

Passidomo, Catarina

Pavloudi, Alexandra

Pearce, John A.

Peplow, Daniel

Pereira, Dora

Pérez, Carmelo García

Pérez-Fuentes, María Del Carmen

Perez-Vaisvidovsky, Nadav

Perikos, Isidoros

Perkins, Krystal

Pernilla, Myrne

Peterson, Jordan Carr

Pettibone, Lisa

PEVCIN, Primož

Philbin, Simon

Pietriková, Emília

Piguet, Etienne

Pikora, Terri

Piña, Olga Duarte

Piva, Mariacristina

Platis, Magdalena

Plouffe, Michael

Poché, Justin D. 
Pop, Izabela Luiza

Popescu, Madalina Ecaterina

Porrovecchio, Alessandro

Postuvan, Vita

Potluka, Oto

Poynting, Scott

Prior, Sarah

Procentese, Fortuna

Progano, Ricardo Nicolas

Progler, Joseph

Pustelnikovaite, Toma

Pyrhönen, Niko

Pyżalski, Jacek

Qasim, Salma

Quam-Wickham, Nancy

Quinn, Camille

Quintana-Murci, Elena

Racolța-Paina, Nicoleta Dorina

Rahman, Osmud

Rahoi-Gilchrest, Rita

Rapoport, Anatoli

Ratajczak, Magdalena

Rawle, Steven

Reddy, Ravi Krishnan

Reder, Alfredo

Rees, Gethin

Reese, Laura A.

Regueiro, Bibiana

Reinertsen, Anne Beate

Reis, Pedro

Relinque, Fernando

Rennels, Jennifer L.

Requena, Pablo E.

Restrepo Baena, Oscar Jaime

Rhodes, Diane McDaniel

Rhodes, Trisha

Ricciardelli, Lauren A.

Richert-Kaźmierska, Anita

Ristić Trajković, Jelena

Rizi, Majid Haghani

Roberts, Ken

Roca-Cuberes, Carles

Rodrigues, Donizete

Rodríguez Jiménez, Carmen

Rodríguez-Ferrándiz, Raúl

Rodriguez-Modroño, Paula

Rogobete, Silviu

Rogozea, Liliana

Rolf, Poulsen

Roman, Caterina

Romero Balsas, Pedro Manuel
Romero Martínez, Ángel

Ronsivalle, Daniele

Rosasco, Paolo

Rosati, Fausta

Rosich, Gina R.

Rössel, Jörg

Rotondo, Francesco

Rumble, Hannah

Rung, Daile

Rutecka-Góra, Joanna

Sabina, Źróbek

Sacramento, Octávio

Sadouni, Samadia

Sæle, Rannveig Grøm

Sainz-de-Baranda, Clara

Salamońska, Justyna

Salazar, Debra J.

Salmeron Manzano, Esther

Sampermans, Dorien

Sampson, Helen

San Pedro Veledo, María Belén

Sánchez-Núñez, Pablo

Sandbæk, Mona

Sandell, Rickard

Šandrk Nukić, Ivana

Sandy, Larissa

Santagati, Mariagrazia

Santana, Andrés

Santha, Agnes

Santos Silva, Dora

Sarapultsev, Alexey

Sarasa-Cabezuelo, Antonio

Sard, Maria

Saura Lacárcel, José Ramón

Schmidt, Eva-Maria

Schmidt, Johanna M.

Schmitt, Leo

Schoppek, Wolfgang

Schubert, Tinka

Schulz, Jay

Schwanebeck, Wieland

Scott, Daniel

Scott, John

Seiz Puyuelo, Marta

Sekulic, Damir

Sercombe, Peter

Serpa, Sandro

Serrano-Montes, José Luis

Seymour, Jack L.

Seymour, Kate

Shacham, Maayan 
Shaver, Frances M.

Shaw, Terry V.

Sheldon, Steven

Sheppard, Paula

Sheu, Guang Yih

Shi, Hon-Yi

Shim, Jae-Mahn

Siddiqui, Nadia

Sidorczuk-Pietraszko, Edyta

Silander, Daniel

Simi, Peter

Simões, Nádia

Smalec, Agnieszka

Smith, Claire

Smyth, Paul

Solange Munoz, Isabel

Solovieva, Yulia

Soverchia, Michela

Spencer, Leland G.

Spiegelhalter, Kate

Stahler-Sholk, Richard

Stankiewicz-Mróz, Anna

Stehle, Maria

Stephan, Johannes

Stojčić, Nebojša

Strauss, Susan

Strielkowski, Wadim

Sundin, Ebba

Sundström, Aksel

Sutter, Glenn C.

Sutton, Jill

Sylvia IV, J.J.

Syrný, Marek

Szymaniec, Piotr

Talavera, Marta

Talukder, Byomkesh

Taylor, Zack

Taylor-Dunn, Holly

Taylor-Smith, Ella

Te Nijenhuis, Jan

Teixeira Domingues, José Pedro

Teixidó, Laura Salamero

Teodora-Mihaela, Iconomescu

Thakor, Mitali

Theorell, Tores

Theurich, Melissa A.

Thomas, Cate

Thomas, Minnie

Thomas, Roger

Thulemark, Maria

Tissot, Sylvie
Tiziana, Mancini

Tomczyk, Łukasz

Tong, Ruipeng

Țoniș Bucea-Manea, Rocsana

Topa, Gabriela

Topidi, Kyriaki

Tracey, Skillington

Trachsel, Mary

Tragaki, Alexandra

Trahar, Sheila

Trygg, Kristina

Tsai, Yao-Te

Tubis, Agnieszka Anna

Tufis, Claudiu D.

Turner, Denise

Twamley, Katherine

Tyburski, Ernest

Uba, Katrin

Uppal, Charu

Vaccarelli, Alessandro

Van Belle, Douglas

Van Hoof, Anita

Vande Walle, Gudrun

Vaughn, Allison

Vaz De Carvalho, Carlos

Vecchiato, Alessandro

Vega, Diego

Veglis, Andreas

Veiga, Ivo

Verhine, Robert

Vik, Tennley

Vila, Natalia

Villar, Oliver

Vincent, Sharon

Violante, Maria Grazia

Visvizi, Anna

Vitale, Tommaso

Voinea, Lelia

Voroshilova, Anzhelika

Wadley, Susan Snow

Wang, Chung-Jen

Wasileski, Gabriela

Watts, Rob William

Weaving, Charlene

Wilkinson, Larrell L.

Wilson, Tom

Wood, Elizabeth

Wroblewski, Angela

WU, Qiaobing

Wyatt, Tanya

Wydra, Doris 
Wylie, Diana

Wynn, Colleen

Xiaoyan, Huang

$\mathrm{Xu}$, Yanfeng

Yam, Shing-Chung Jonathan

Yates, Miriam

Yllo, Kersti Alice

Young, Michael

Zahariadis, Nikolaos

Zajda, Joseph
Zambrana, Ivis Garcia

Zare, Hossein

Zhang, Hui

Zhang, Xufang

Zhou, Shu

Zhu, Guoniu

Zollner, Hans

Zolnikov, Tara Rava

Žulec, Mirna 